\title{
Experiment of Event Injection Technology for Network Dependability Evaluation
}

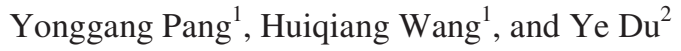 \\ ${ }^{1}$ College of Computer Science and Technology, \\ Harbin Engeering University 150001 Harbin, China \\ pangyonggang@hrbeu .edu.cn, \\ wanghuiqiang@hrbeu.edu.cn \\ ${ }^{2}$ College of Computer, Beijing Jiaotong University, 100044 Beijing, China \\ mail_dy@163.com
}

\begin{abstract}
Dependability evaluation of computer network is an integrated and complex research problem. Evolving from fault injection, event injection can be better used to evaluate dependability of computer network system. According to the principle of event injection, an experiment model for network dependability evaluation was designed and correlative experiments were carried out. At last, application of event injection for dependability evaluation on computer network was discussed.
\end{abstract}

Keywords: dependability evaluation; event injection; DDoS; Loadrunner.

\section{Introduction}

In some important domains, such as national defense, avigation, finance and transportation, dependability of its computer network system is an important problem. Dependability of a system is related to network reliability, availability, security and safety, so how to evaluate the integrated dependability is a problem to be solved urgently [1]. Nowadays, the research on dependability of computer network system concludes important server system, small-size network and web application network, and the conception of dependability is still developing. However, method, model and relative index system of dependability evaluation are lacking, research on these domains sill need to be further carried on.

Event injection technology is introduced to the research on dependability evaluation of network system, by which the integrated dependability of a target system can be tested [2]. Event injection technology adapts better to the experiment in the computer network environment than fault injection. In the event injection experiment, relative characteristic events can be injected into the system to check its reaction according to the relative attributes and characters of dependability evaluation. This paper sequentially dissertates the conception of event injection technology, some event injection experiments and integrated judgment to the experiments. At last, some problems of event injection technology in network experiment are discussed. 


\section{Dependability of Computer Network}

The new conception of dependability[3] was formally advanced by J.C.Laprie in 1985, which included conceptions of reliability, availability, maintainability and safety at that time, that is to say, the reliable computing and fault-tolerance were joined into the conception of dependability. From the medium term of 1980s', fault-tolerance technology was applied to deal with the security threat and a series of exploring researches on hostile failure problems led to a fusion of the two conceptions of security and dependable computing. After that, deep and close discussion of some experts caused the appearance of an academic patent which has further effect [4]. After that, J.C.Laprie deeper illustrated his viewpoint many times at different occasions. After 1995, with the worsening of Internet security, together with the developing of security technologies and changing of attack means, people had to afresh scan the essence of dependable computing and security computing. And the new consideration of dependable computing and security computing by A.Avizienis, J.C.Laprie, Brian Randell, and Carl Landwehr reflected the newest changes.

Correlative conceptions including trusted computing from U.S. military [5] and trustworthy computing advanced by Bill Gates of Microsoft developed. During research on dependability [6], correlative conceptions are developing continuously, but there isn't uniform standard.

\section{Event Injection Model}

During the dependability evaluation on system, there were not only traditional exact models such as the network hardware reliability problems, but also the non-accurate models, such as the security problem, so event injection evolving from fault injection can solve these problems better.

Event injection technology is an experimental process, in which different kinds of system events including reliability events, availability events, security events of network system are injected into the target system, at the same time, response information of injected events is analyzed or called back by corresponding system, and at last relative results are provided to experimenters. Key technologies affecting the dependability evaluation result by event injection technology mainly are: selection of event model library, statistical event coverage, and experiment comprehensive evaluation.

\subsection{Selection of Event Model Library}

Event model library should include the events that can influence the service ability of the target system. The injected event model selected is more similar to the events occurred during the actual running of the system or covers them to the greatest extent, the results of the experiment will be more accurate. A suitable event model library should follow two principles: Firstly, it should include a majority of event models that can influence the actual running of the system and cause performance decline of the target computer systems; Secondly, event models in it can detect the vulnerability of the target system. 


\subsection{Statistical Event Coverage}

The events injected into an running system can't include all of the events faced by system applications and injection strategy of one event can't represent entire instances, at the same time, harm extents of different events faced by system are different from each other, so during the dependability evaluation on the target system, the satisfactory degree which is named as statistical event coverage should have a credibility interval.

\subsection{Experiment Comprehensive Evaluation}

Computer network dependability evaluation includes reliability, availability, safety and security, and so on, moreover the network system can be divided into local area network, distributed network and internet, therefore the network system dependability research is very broad, the corresponding test data will be massive, how to obtain a system' dependability degree is a complex question.

\section{Event Injection Experiment}

In the network dependability evaluation, the network service performance and security are very important. This article simulates and evaluates the web server in network using event injection technology, use Loadrunner to test the basic performance of the network and use the DDoS attack test to influence the system.

This experiment is to test the operating performance of the target system, LoadRunner8.0 test software is used. The results can then be analyzed in details, to explore the reasons for particular behaviour. the data in Table 1 will be used as the index data in the dependability evaluation to the target server system.

Table 1. Main tesing data of web sever

\begin{tabular}{c|c|c|c|c}
\hline Measure events & index & $\min$ & $\max$ & average \\
\hline connection & Connection shutdowns & 0.125 & 83.063 & 42.497 \\
\cline { 2 - 5 } rate & New connections & 0.107 & 83.125 & 42.563 \\
\hline error rate & Error-26628 & 0 & 5.438 & 2.745 \\
\hline Hits per Sec & Hits/s & 0.286 & 153.063 & 75.377 \\
\hline HTTP Re- & HTTP_200 & 0.143 & 91.844 & 41.775 \\
\cline { 2 - 5 } sponses per & HTTP_403 & 0 & 75.75 & 33.291 \\
\cline { 2 - 5 } Sec_ & HTTP_404 & 0 & 0.625 & 0.311 \\
\hline flow speed & B/s & 2313 & 591651 & 340855 \\
\hline
\end{tabular}

DDoS makes the failure of attacked network server. In Figure 1 and Figure 2, the server average response time increases along with the attack flow increase, the mean flow speed reduces along with the attack flow increase. At the same time, along with the attack duration extension, the server average response time increases, mean flow speed reduces. When the attack computer increases, the server response 


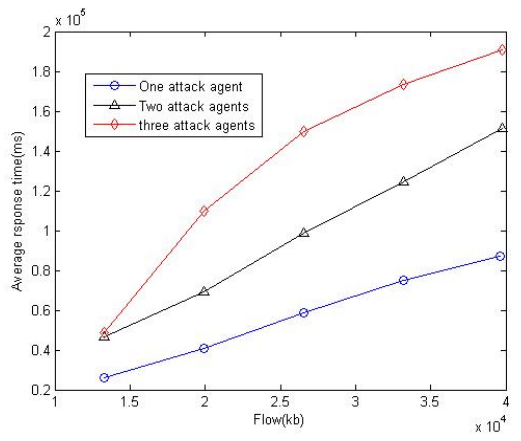

Fig. 1. Average response time curves of server

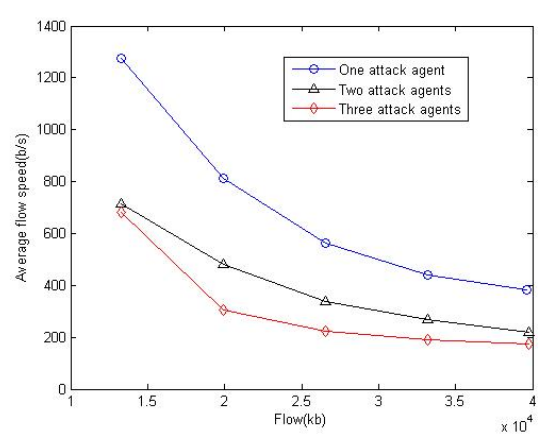

Fig. 2. Average flow speed curves of server

time obviously increases, with the server processing attack request event increases, the server response slows down even in the same flow situation.

\section{Conclusions}

In the experiment method, event injection technology can preferably solve the problems of network dependability evaluation. Event injection technology can not only analyze the cases when the target system fail but also analyze the cases when the capability of target system performance declines or the system not being able to offer satisfied services to users.

\section{References}

1. Yves Deswarte, Karama Kanoun, Jeam-Claude Laprie. Diversity against accidental and deliberate faults. PRDC'99,Hong Kong, 1999.

2. Huiqiang Wang, Yonggang Pang, Ye Du. Evaluation of network dependability using event injection. In: Proc of the APWeb 2006 Int'1 Workshop: XRA, IWSN, MEGA, and ICSE. Berlin: Springer, (2006) 991-998.

3. J.C. Laprie. Dependable Computing and Fault Tolerance: Concepts and Terminology. Proc.15th IEEE Int'1 Symp. Fault-Tolerant Computing (FTCS-15), (1985) 2-11.

4. J.C. Laprie, ed. Dependability: basic concepts and terminology-in English, French, German, Italian and Japanese. In Dependable Computing and Fault Tolerance. Springer-Verlag, Vienna, (1992) 265.

5. Department of defense trusted computer system evaluation criteria. http://www.radium.ncsc.mil/ tpep/library/rainbow/5200.28-STD.html.

6. Bill Gates. Trustworthy Computing, 2002. 